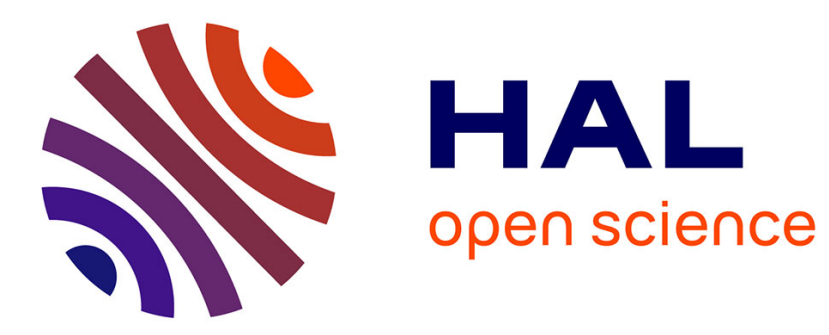

\title{
Des interactions sociales en formation universitaire à distance: une approche microsociologique exploratoire et inférentielle
}

Jean-Claude Regnier, Annick Pradeau

\section{- To cite this version:}

Jean-Claude Regnier, Annick Pradeau. Des interactions sociales en formation universitaire à distance : une approche microsociologique exploratoire et inférentielle. STICEF (Sciences et Technologies de l'Information et de la Communication pour l'Éducation et la Formation), 2009, 16, 20 p. hal-00696348

\section{HAL Id: hal-00696348 \\ https://hal.science/hal-00696348}

Submitted on 11 May 2012

HAL is a multi-disciplinary open access archive for the deposit and dissemination of scientific research documents, whether they are published or not. The documents may come from teaching and research institutions in France or abroad, or from public or private research centers.
L'archive ouverte pluridisciplinaire HAL, est destinée au dépôt et à la diffusion de documents scientifiques de niveau recherche, publiés ou non, émanant des établissements d'enseignement et de recherche français ou étrangers, des laboratoires publics ou privés. 


\title{
Des interactions sociales en formation universitaire à distance, Une approche microsociologique exploratoire et inférentielle
}

\author{
Jean-Claude REGNIER*, Annick PRADEAU** ${ }^{* *}$ Université de Lyon UMR 5191 \\ ICAR, ${ }^{* *}$ Université de Rouen)]
}

- RÉSUMÉ : Dans cet article, nous nous intéressons à l'organisation des interactions sociales dans un dispositif de formation universitaire en ligne. Les hypothèses présument que, malgré la distance entre les étudiants, les interactions sont riches et structurées. Les contraintes techniques et les caractéristiques spatio-temporelles influent sur les acteurs qui agissent sur l'organisation sociale. Le champ théorique est celui de la sociologie interactionniste d'Erving Goffman. La méthodologie consiste à observer les traces des échanges entre six étudiants engagés dans un apprentissage collaboratif sur un forum de discussion du campus numérique FORSE. La dimension exploratoire est complétée d'une démarche inférentielle consistant à examiner les concepts goffmaniens pour considérer leur congruence au champ spécifique des interactions sans coprésence physique. Nous proposons d'assimiler cet environnement numérique à un nouveau cadre de l'expérience où s'instaure un ordre interactionnel qu'il s'agit de mieux comprendre.

- MOTS CLÉS : Interactions sociales, apprentissage collaboratif, campus numérique, forum de discussion, sociologie interactionniste

- ABSTRACT : This paper focuses on the organization of social interaction in an online training of university students. It was supposed that despite the distance between students, mutual interactions are rich and structured. Technical constraints and spatio-temporal characteristics influence on participants who participate in social interaction. The theoretical framework of this study is based on the theory of Erving Goffman. The traces of exchanges between six students engaged in collaborative learning on a forum for discussion of FORSE campus were observed. The exploratory dimension is complemented by an inferential process of reviewing the Goffmanian concepts to consider matching of specific field of interaction without physical copresence of participants. We propose to treat this digital environment to a new frame of experience where an interactional order is established which is better understood.

KEYWORDS : Social interactions, collaborative learning, digital campus, electronic discussion group, inter-actionist sociology

- 1 . Introduction

- 2. Cadre conceptuel : microsociologie et apprentissage à distance

- 3 . Méthodologie : observation de type naturaliste d'un forum de discussion

- 4. Premiers résultats de l'observation du forum

- $\underline{5}$. Apprentissage collaboratif à distance et concepts goffmaniens

$\circ \underline{6 . \text { Conclusion }}$

- $\underline{7 . \text { Références }}$

$\circ \underline{8 . ~ A N N E X E S}$

\section{Introduction}

Internet représente un immense territoire en expansion accélérée (Levy, 1997) qui soulève des questions d'ordre sociétal. La réalité est augmentée d'un nouvel état du réel (Quéau, 2000), qui offre des moyens d'échanges inédits. Ces évolutions ne sont pas sans incidences sur le monde de l'éducation. Notre propos s'intéresse à la question de l'organisation sociale dans le contexte particulier d'une formation universitaire en ligne que nous avons abordée dans le cadre de travaux scientifiques universitaires (Pradeau, 2009). 
Parmi diverses questions, nous proposons d'interroger l'organisation des relations qui s'établissent entre les étudiants travaillant en groupe dans le cadre d'un cours, en l'occurrence de statistique et méthodes qualitatives et quantitatives au niveau du diplôme de Master 1 en sciences de l'éducation. Nous sommes partis du présupposé que les interactions sont riches et structurées, et que les contraintes techniques et spatio-temporelles ont des effets conséquents sur les pratiques sociales mêmes. Dans une seconde étape faisant suite à celle-ci dont nous ne rendrons pas compte ici, nous nous sommes engagés dans l'identification et la mesure des effets des interactions produites au sein des situations organisées par cette ingénierie pédagogique sur la conceptualisation en statistique et les performances des sujets. Nous nous limitons à aborder ces interactions entre pairs en nous appuyant sur quelques concepts théoriques développés par le sociologue Erving Goffman. Notre choix méthodologique a été de mener une observation de type naturaliste des messages enregistrés sur un forum de discussion dédié à un groupe de six étudiants participant à un travail collaboratif à visée formative et évaluative sur le campus numérique FORSE ${ }^{1}$. Comment s'organise cette petite unité sociale agissant et apprenant sans coprésence physique et quelles en sont les caractéristiques? Rendre visible et lisible ce que ces apprenants échangent sans se voir, participe d'une meilleure connaissance de l'organisation de leurs interactions et d'une certaine manière d'une meilleure compréhension des conditions d'efficience d'une situation d'enseignement et d'apprentissage en ligne. L'importance de la dimension sociale dans un contexte d'apprentissage est une notion ancienne, largement développée par les théories socio-constructivistes. Ainsi les travaux conduits dans les années 70-80, sur le concept de conflit socio-cognitif (Perret-Clermont, 1986) ont mis en évidence la dimension sociale du développement de l'intelligence et le rôle des interactions dans le développement cognitif individuel. La situation pédagogique que nous prenons comme base d'observation a d'ailleurs été construite par l'enseignant responsable de ce cours à partir de ce présupposé théorique (voir fiche pédagogique présentée en annexe). Plus généralement nous pouvons affirmer que la question du travail en groupe pour apprendre se trouve actualisée par la multiplication des espaces virtuels de formation. L'espace d'échanges étudié, un forum de discussion, se constitue en un lieu de formalisation et de conservation des interactions, qu'il s'est agi d'observer, qualifier et quantifier pour tenter de comprendre ce qui se joue dans cet espace de formation.

\section{Cadre conceptuel : microsociologie et apprentissage à distance}

Le choix d'un point de vue théorique est une nécessité qui nous conduit à privilégier celui proposé par Erving Goffman (1922-1982) pour lequel l'échange entre deux individus est considéré comme la relation sociale la plus élémentaire.

\subsection{Les interactions sociales selon le point de vue de Erving Goffman}

Grâce à un regard microsociologique porté à l'intérieur des situations sociales, cet explorateur des situations ordinaires a construit une sorte de "grammaire de l'interaction" (Blandin, 2004) (p.362). Il définissait les contacts entre des personnes "comme toute occasion où un individu parvient à la portée de la réponse d'un autre, que ce soit par coprésence physique, par connexion téléphonique ou par échange épistolaire" (Goffman, 1988) (p.202). Cette définition confirme le sens qu'il donne au contact social, qu'il lie avant tout à la possible réponse de l'autre, nuançant ainsi l'idée d'une nécessaire coprésence immédiate. S'il avait vécu le développement d'Internet des années 90, il aurait sans doute pu étendre ses recherches aux communications par messagerie électronique ou forum de discussion. Dans sa vision du monde, le sujet et la structure sont intimement reliés par l'interaction, qui tout à la fois individualise et socialise (Bonicco, 2007) (p.31). Il établit ainsi la jonction entre le "micro" et le "macro" postulant que l'étude des interactions au sein d'un petit groupe permet d'appréhender la société tout entière (Winkin. 2001) (p.123). Il s'est attaché dans toute son œuvre à poser une loupe sur les détails de la vie sociale

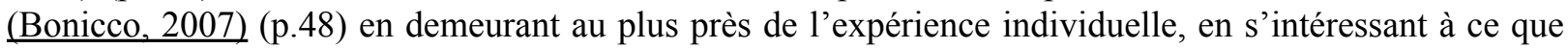
font les acteurs plutôt qu'à ce qu'ils pensent ou disent penser.

Goffman a théorisé l'interaction en développant une série de concepts originaux utilisant une terminologie dramaturgique : acteur, rôle, décor, masque, scène, coulisses, représentation, interprétation, mise en scène, etc. (Goffman, 1973a). Dans toute rencontre, directe ou médiatisée, l'individu extériorise un certain nombre d'actes qui donnent une interprétation de lui-même. La face est le masque que présente 
l'acteur social à ses partenaires dans l'interaction. Cette image est conditionnée par ce que l'autre attend dans la situation du moment. Pour analyser les situations sociales, il définit des régions, la scène et les coulisses (Goffman, 1973a) (p.131), dans lesquelles l'acteur serait plus ou moins exposé au regard ou à la présence des autres. Ce partage physique entre les espaces de représentation détermine la façon dont chaque rôle est joué par les personnes en interactions. En changeant de lieu un individu modifie ses attitudes et actions en fonction du nouveau cadre de son expérience. Sociologue mais aussi linguiste, Goffman s'est en outre intéressé à la conversation comme modèle de l'interaction. Il différencie les échanges confirmatifs et les échanges réparateurs (Goffman, 1973b) (p.74). Les premiers visent à entretenir ou à confirmer une relation établie. L'exemple le plus courant en est l'échange de salutations. Les seconds servent à restaurer l'équilibre interactionnel lorsqu'il est mis en péril par un acteur (Joseph, 1998) (p.109).

Selon Goffman, une équipe est "un ensemble de personnes dont la coopération très étroite est indispensable au maintien d'une définition donnée de la situation" (Goffman, 1973a) (p.102). De même que l'individu, l'équipe est en représentation. Une relation d'interdépendance se crée entre ses membres, chacun devant faire confiance aux autres quant à leur bonne conduite pour le maintien de l'ordre interactionnel en cours. Il évoque la tendance dans une équipe à la création de liens de familiarité et de loyauté de chacun envers ses équipiers (Goffman, 1973a) (p.84). Cette obligation sociale de marquer son engagement peut varier pour chacun des acteurs selon ses obligations sur d'autres scènes (Joseph, 1998) (p.123).

Nous avons présenté quelques concepts théoriques extraits des écrits d'Erving Goffman et d'auteurs qui ont analysé son oeuvre. Nous allons maintenant aborder la notion de formation à distance à l'heure des Technologies de l'Information et de la Communication (TIC) en présentant certaines de ses spécificités.

\subsection{La formation à distance et les technologies de l'information et de la communication}

Aussi ancienne que l'invention du timbre poste (1840), la formation à distance (FAD) s'est transformée au gré des évolutions technologiques. Internet facilite la mise en relation d'individus ou groupes éloignés géographiquement. Les organismes de formation ont ainsi pu étendre leurs prestations à ce nouvel espace dans un processus de migration d'un modèle basé sur le télé-enseignement avec des cours papiers vers un dispositif en ligne, comme ce fut le cas pour le campus FORSE (Wallet, 2007) (p.9). Le progrès technique s'accompagne d'une modification des pratiques sociales, avec en particulier la multiplication des réseaux sociaux. Des pages statiques aux pages dynamiques, l'usage de l'Internet s'est orienté vers des supports et des pratiques où la notion d'interaction prend tout son sens. L'internaute n'est plus un simple spectateur mais il devient acteur, interagissant avec d'autres utilisateurs et échangeant toutes sortes de données. Le passage de la connexion technologique à la communication humaine ne va cependant pas de soi, et être interconnectés ne signifie pas nécessairement être en relation (Wolton, 2000) (p.107).

Les faits d'éducation ne peuvent échapper à ces évolutions techniques et sociales. En France, au début des années 2000, plusieurs appels à projets des Ministères de l'Education et de la Recherche conduisent au développement et à la labellisation de campus numériques (Averouz et Touzot, 2002), (Fichez, 2006). Des formations hybrides mixent des temps de formation en face-à-face et à distance, considérant ces modalités d'enseignement et d'apprentissage comme complémentaires et non antagoniques (Glikman, 2002). Le Campus numérique FORSE est l'un de ces campus labellisés en réponse à l'appel à projets "campus numériques" de 2001. En 2002, le troisième appel à projet concernant les campus numériques se complète d'une proposition de création d'Environnements Numériques de Travail (ENT) qui permettent, par le biais de dispositifs techniques, de mettre de nouveaux services à disposition des acteurs de ces campus. Les ENT se matérialisent par des plates-formes où sont regroupés les outils et ressources pédagogiques nécessaires à leurs utilisateurs. Selon Audran, "le principe de ces plates-formes doit être non de présenter des contenus, mais de créer des "situations" propres à déclencher les interactions /.../ Interagir avec des personnes qui sont hors du champ de perception direct demande un apprentissage qui ne va pas de soi" (Audran, 2006) (p.37). Ces ENT ne correspondent donc pas seulement à l'extension de modalités de formation existantes mais permettent la construction d'un nouveau modèle de relations sociales entre les différents acteurs de la formation universitaire, tant des enseignants et des apprenants que des personnels administratifs et techniques. Le campus ne se trouve plus dans un espace géographique déterminé, mais sur un serveur, dans l'état d'un espace numérique. Les unités de lieu, de 
temps, d'action, qui caractérisent la salle de classe dans l'enseignement traditionnel sont bouleversées (Glikman, 2002) et conduisent à une nécessaire redéfinition de l'espace éducatif et de ses composants pédagogiques.

Des apprenants, situés dans des lieux géographiques différents, peuvent se retrouver pour étudier ensemble sur un même espace virtuel tel qu'un forum de discussion, défini par Mangenot comme "un dispositif technique et un lieu d'interactions sociales qui se caractérise par une communication écrite, asynchrone, publique et structurée" (Mangenot, 2008). Cette situation dans laquelle deux ou plusieurs personnes apprennent ou essaient d'apprendre ensemble (Dillenbourg, 1999) est étendue aux espaces virtuels de formation. C'est le cas du campus FORSE dont le modèle pédagogique au niveau Master insiste beaucoup sur le travail collaboratif (Wallet, 2007) (p.11), i.e. l'élaboration d'une production collective en partageant des ressources par le biais d'espaces de communication virtuels. La possibilité technique d'interagir n'est cependant pas suffisante pour qu'une démarche d'apprentissage soit efficiente. "Collaborer ne se décrète pas mais se régule" (Wallet, 2004). La structure et la qualité des échanges déterminent le déroulement du processus de même que la prise en compte du versant pédagogique. En effet, ces pratiques collaboratives en ligne restent assez éloignées de celles vécues en face-à-face, ce qui nécessite une adaptation de la part des différents acteurs et le développement de compétences particulières (Bruillard, 2008). "Travailler ou apprendre en groupe suppose de mobiliser un certain nombre de compétences sociales, préalablement nécessaires à la réalisation de la tâche et/ou susceptibles de se développer tout au long de la situation collaborative" (Simeone et al., 2007).

Malgré la prolifération de vidéos et d'images sur le Web, le rapport au texte écrit en langue naturelle reste une préoccupation centrale au sein des campus numériques, comme dans l'ensemble du secteur éducatif. Cette communication par l'écrit limite les indices socio-émotifs et comportementaux (regard, posture, voix,...) présents dans la relation en face-à-face. Tholozan va jusqu'à considérer que l'écrit informatique, souvent condensé, peut être froid et doit être "humainement réchauffé" (Tholozan, 2004) (p.5). L'utilisation de codes spécifiques, tels que les émoticônes (smileys) ou l'écriture en majuscule pour renforcer le propos, permet de donner au message sa tonalité émotionnelle visant à faciliter ainsi l'interprétation du texte transmis. A ce mode de communication spécifique s'ajoute une dimension temporelle car une grande partie des échanges se fait sur des forums de discussion ou par messagerie électronique, autrement dit sur un mode asynchrone. Par ailleurs, les écrits, enregistrés sur le support numérique et consultables à tout moment, sont comme une mémoire du groupe d'acteurs. Pour Arino, nous assistons à la naissance d'une nouvelle entité spatio-temporelle, un "nouveau territoire des relations sociales", marqué par la complexité et par la multiplication des cadres de l'expérience au sens défini par Goffman $\underline{(\text { Arino, 2001). }}$.

L'enseignement traditionnel suppose la co-présence physique des acteurs : enseignants et apprenants dans un même lieu. En formation en ligne, cette proximité physique peut être inexistante, ou se produire de manière ponctuelle, lors de regroupements dans les locaux de l'université. Ce cadre de l'expérience modifie la perception de l'autre dans l'interaction. Jacquinot-Delaunay a développé la notion de sentiment de présence perçu différemment suivant les individus, en soulignant que "la présence physique est une des dimensions de la présence et qu'il y a tout un éventail de présences qui vont de l'absence totale à la coprésence" (Jacquinot-Delaunay, 2001). La notion de distance, très subjective, serait avant tout une construction mentale, un sentiment, sans doute sur un autre registre, mais du même ordre que celui de

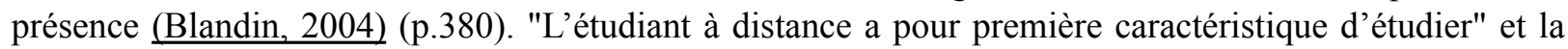
distance représente une modalité particulière dans ce contexte d'apprentissage et probablement pour certains étudiants une difficulté supplémentaire pour suivre un cursus (Béziat et Wallet, 2007) (p.73). Se rendre visible et perceptible à distance est une façon de s'incarner dans le cyberespace. L'interaction à distance nécessite donc de multiplier les divers moyens de "véhiculer les signes de la présence" (Weissberg, 2001). Le renforcement de ce "sentiment de présence" est sans doute nécessaire pour dépasser ce que Wolton a appelé les "solitudes interactives" (Wolton, 2000) (p.106), et peut-être limiter le nombre particulièrement important d'abandons dans les dispositifs de formation à distance.

\section{Méthodologie : observation de type naturaliste d'un forum de discussion}

L'approche méthodologique est plurielle, avec d'une part un versant déductif dans le sens d'une 
vérification de l'aspect structuré et ritualisé des interactions, et d'autre part un versant inductif consistant à donner du sens à ce qui émerge des données à la lumière des concepts interactionnistes, d'autre part encore, un versant abductif, dans la mesure où nous avons eu parfois recours à une sorte d'intuition créative "creative insight", (Peirce, 1903, 2006) pour expliciter des hypothèses plausibles pour comprendre ou expliquer certains phénomènes qui nous ont paru surprenants. Dans sa proposition de classification des recherches sur les Technologies de l'Information et de la Communication, Wallet considère que "l'approche inductive se construit essentiellement par des observations de pratiques des acteurs dans les lieux de formation ou par l'usage des méthodologies quantitatives ou qualitatives inscrites dans les sciences humaines" (Wallet, 2002). Dans sa typologie des recherches sur les forums de discussion, Bruillard évoque les études qui tirent partie des données d'interaction que l'on peut "extraire et traiter, sans perturber les communautés qui échangent" (Bruillard, 2005). L'observation des traces disponibles focalisées sur l'activité permet de comprendre ce qui se joue dans les interactions afin que les enseignants et apprenants régulent au mieux les activités censées favoriser les apprentissages (Bruillard. $\underline{2008)}$ (p.57). Nous nous situons sur ce modèle exploratoire. Rappelons par ailleurs que l'observation sur le terrain a été largement valorisée par l'Ecole de Chicago dont Goffman, parmi d'autres, a renouvelé le contenu empirique et théorique.

Pour rester sur ce registre, nous avons donc examiné les messages enregistrés sur un forum de discussion, traces écrites indicatrices des interactions entre les étudiants. Ce type de données permet un rapprochement entre construction de données par observation et analyse de contenu (Blanchet et al., 2000) (p.59). Cette forme d'observation a posteriori, et donc non participante, peut éviter les effets induits par la présence de l'observateur sur la situation observée, tout en sachant que d'autres informations plus directement liées au vécu dynamique de l'activité nous échappent. D'un autre point de vue, nous pourrions considérer que les fichiers $\log$ ou autres portant les traces des échanges via des connexions numériques, constituent des documents dont l'analyse peut être aidée par les techniques de l'analyse documentaire. Nous conduisons cette analyse et recherche de sens dans notre cadre théorique qui prend appui, comme nous l'avons présenté plus haut, sur un domaine conceptuel développé par Goffman. Cette extension d'une théorie existante à un champ plus large, ici les interactions sociales en dehors d'une coprésence physique, est définie par Van Der Maren comme une analyse inférentielle (Van Der Maren, 1995) (p.149).

Les données construites et présentées dans (Pradeau, 2009) (p. 95-150) sont extraites des traces laissées sur la plate-forme du campus numérique FORSE. Le choix a été fait de focaliser notre observation sur un forum de discussion proposé aux étudiants d'une promotion de Master1 en Sciences de l'Education (année 2006-2007).

A ce niveau de la recherche, le corpus construit puis analysé est constitué des messages échangés par un groupe comprenant quatre étudiantes et deux étudiants participant à un travail collaboratif dans le cadre d'un cours de méthodologie: méthodes quantitatives, méthodes qualitatives et statistique pour la recherche en sciences de l'éducation. Le choix de cet échantillon est guidé par le fait que les sujets offrent quelques caractéristiques prototypiques au sens de (Rosch, 1975). Comme nous le rapportons en annexe, une fiche-guide explicitait les objectifs et les attentes pédagogiques. Cette fiche était communiquée et commentée aux étudiants lors du regroupement de septembre par l'enseignant $\stackrel{2}{-}$ responsable du cours et le tuteur, animateur de plate-forme, chargé de superviser l'organisation du travail de ce cours. Synthétiquement, il s'agit de compléter la formation méthodologique acquise en licence en particulier dans le domaine des méthodes quantitatives [A], méthodes qualitatives [B] et de la statistique et d'aider à la mise en lien des acquis méthodologiques dans le cadre de la recherche conduite pour le mémoire de Master1 (Maîtrise). Pour atteindre cet objectif, une situation problème est proposée autour d'un thème global commun : la réussite scolaire, prétexte à la mise en œuvre des outils et méthodes visées dans la formation. Ce thème est décliné en quatre sous-thèmes : Réussite scolaire et climat familial, genre, travail en équipe pédagogique, classes sociales, à partir desquels se sont constitués les 8 groupes parmi les 45 étudiants inscrits dans la formation. Les tâches prescrites sont à la fois d'ordre individuel et d'ordre collectif. Ces travaux sont évalués et intégrés à l'évaluation globale pour l'obtention du diplôme de Maîtrise.

Nous avons extrait les traces enregistrées sur l'un des huit forums dédiés aux groupes d'étudiants. Ces données invoquées (Van Der Maren, 1995) ont ensuite fait l'objet d'une analyse de contenu. L'anonymisation et le codage des données se sont réalisés par l'attribution aux interactants de prénoms 
fictifs dont les initiales correspondent au codage A, B, C, D, E, F pour les 6 étudiants travaillant ensemble et G, H, I, pour les intervenants extérieurs au groupe, soit Ariane, Bernard, Carole, Denise, Emile, Fabienne et Geneviève, Hermine, Isidore. Pour transformer les données brutes en données organisées, des catégorisations par découpage du corpus initial ont permis d'obtenir de nouvelles données. Les fils de discussion sont codés "Dn". Le premier fil est ainsi intitulé D1, le dernier D56. Sur le même principe, les messages isolés, c'est-à-dire ceux qui n'ont pas obtenu de réponse, sont codés "In". Dans chaque fil de discussion les messages sont codés "Mn" où $n$ est le numéro du message sur le forum. Par exemple, nous avons noté D1M540 pour faire référence au message intitulé 540 du premier fil de discussion. Des analyses mois par mois ont permis des comparaisons entre les différents éléments observés. Afin de dégager du sens du contenu des messages, ainsi que pour en évaluer l'aspect évolutif, nous avons procédé à une observation sur trois périodes ainsi qu'à une catégorisation par unité thématique (Bardin, 2007). Chaque phase observée se déroulait sur une période de huit jours en début, en milieu, puis en fin de session (sous-corpus P1, P2 et P3). Pour chaque sous-corpus, le codage correspondant aux unités de sens a été présenté sous forme de matrice des données brutes, afin de faire apparaître la présence ou l'absence d'un élément à des fins descriptives et comparatives.

Cette observation d'un petit groupe d'étudiants est reproductible. Multiplier ce type de démarche sur un même campus ou sur des campus différents pourrait contribuer à faire émerger des constantes ou particularités repérables au sein de petites unités sociales engagées dans une situation d'enseignementapprentissage.

\section{Premiers résultats de l'observation du forum}

L'observation minutieuse des données recueillies permet de faire les constats suivants :

- Sur un plan quantitatif :

- La session de travail collaboratif se déroule sur une période de six mois et demi de septembre à avril (199 jours)

- Le corpus est constitué de trois cent trente neuf messages, dont huit ont été déposés par trois étudiants extérieurs au groupe de travail.

- Cinquante-six fils de discussion, correspondant à un message initiateur et aux messages réponses qui lui succèdent, sont comptabilisés durant la session. Les fils de discussion semblent se dérouler comme des conversations, tandis que vingt-quatre messages restent isolés.

- Le nombre de messages déposés par chaque étudiant met en évidence une grande disparité :

\begin{tabular}{|l||l|l|l|l|l|l||l|l|l|}
\hline Etudiants & A & B & C & D & E & F & G & H & I \\
\hline $\begin{array}{l}\text { Nbre de } \\
\text { messages }\end{array}$ & 114 & 66 & 11 & 47 & 46 & 47 & 5 & 2 & 1 \\
\hline
\end{tabular}

Tableau 1 : nombre de messages déposés par étudiant (Pradeau, 2009) p. 39.

- 110 pièces jointes sont échangées sur le forum (109 déposées par des étudiants du groupe et une par une étudiante extérieure au groupe).

- Sur un plan qualitatif :

- Il existe des actions visibles, comme déposer des messages, répondre à des messages, ajouter des pièces jointes. Les traces de ces actions sont observables à tout moment car mémorisées et constitutives du corpus analysé.

- Il existe aussi des actions non visibles, mais qui sont évoquées dans le contenu des messages, comme les références à des actions extérieures (e.g. se téléphoner, rechercher des informations, se rencontrer sur un espace virtuel pour un clavardage).

- La structuration des messages et des échanges est très marquée : une partie des messages reste sans 
réponse, une autre partie engage des fils de discussion qui sont eux aussi très structurés.

- Le nombre d'interactions visibles est très variable au cours de la session, avec des périodes d'activité intense (mi-octobre et mi-janvier) et d'autres de fort ralentissement de l'action, comme entre le 7 novembre et le 6 décembre, soit une période d'un mois, où aucun message n'est déposé sur le forum.

- Les messages sont à priori adressés à tous les membres du groupe. Dans le cas contraire, l'émetteur précise le destinataire particulier, soit dans la partie introductive, soit dans le corps du message.

- Le contenu des messages apparaît très diversifié avec des salutations, des échanges de connaissances en lien avec le cours ou d'informations (aspect techniques, organisationnels, etc.), des références à des événements extérieurs (clavardages, appels téléphoniques, vie personnelle, etc.), des aspects émotionnels avec en particulier des émoticônes qui peuvent donner aux messages des tonalités affectives significatives.

\subsection{Aspects structurels}

Le développement des forums est soumis à des contraintes techniques qui déterminent leur ossature. La structure en arborescence est un modèle habituel. Dans le cas du forum présenté dans cette étude, il s'agit d'une structure sous forme de liste indentée, c'est-à-dire que les messages s'accrochent les uns aux autres pour chacun des fils de discussion. Différents onglets ou boutons cliquables permettent des actions sur le forum. Les messages qui sont des réponses sont intitulés "Re : titre du message initial". Cet objet du message est lui aussi cliquable. Cette nouvelle action possible participe à la création de la structure en arborescence. Des boutons cliquables permettent d'offrir plusieurs possibilités d'affichage comme le dépliage ou non des fils de discussion, la mise en évidence des messages non lus, la présentation de l'ensemble des messages par ordre chronologique de mise en ligne. Un forum peut être privé ou public. Dans le cas présent, seuls les étudiants inscrits dans la promotion de Master1 sur le campus FORSE ainsi que les enseignants peuvent accéder au forum, voir les messages, les consulter et en publier de nouveaux. Les espaces dédiés aux différents groupes de travail collaboratif sont ouverts à tous les étudiants de la promotion. Malgré cette possibilité, les étudiants extérieurs au groupe n'y interviennent pas ou très peu. En arrivant sur le forum, l'étudiant ne prend pas connaissance de façon automatique du contenu des messages. Une démarche volontaire est nécessaire. L'action préalable à la lecture des contenus est l'ouverture du message par un clic sur son objet. Ce mode de lecture par navigation induit plusieurs chemins ou parcours de lecture possibles, et donc différents accès aux informations contenues dans les messages. Un étudiant qui ouvre et prend connaissance de tous les messages n'a pas le même comportement interactionnel que celui qui n'en ouvre qu'une partie, quelle qu'en soit la raison.

Cinquante-six (56) messages initiateurs démarrent des fils de discussions. Le fait de déposer un message suppose en effet plusieurs réactions possibles de la part des autres étudiants :

- soit aucune action lorsque l'arrivée d'un message est constatée sans qu'il ne soit ouvert,

- soit une ouverture du message pour le lire mais sans y répondre,

- soit une ouverture pour une lecture suivie de la rédaction et l'envoi d'une réponse. L'expédition du message peut être différée puisqu'il existe une fonction permettant d'enregistrer un message comme "brouillon".

Le plus grand nombre de fils de discussion, comparativement aux messages isolés, souligne la tendance globale à l'enchaînement d'actions entre les différents participants. Dans la majorité des cas, l'action de déposer un message déclenche une autre action sur le même modèle, à savoir l'émission d'un nouveau message. Sur les 339 messages du forum, 259 sont des messages-réponses soit plus des trois quarts ( $76 \%$, du corpus initial). Les messages-réponses ne répondent pas systématiquement au premier message, mais peuvent être une réponse à un autre message-réponse. Chaque fil de discussion s'inscrit dans une unité de temps définie par les dates de publication du premier et du dernier message. Ce temps d'interactions peut être très long, contrairement à un échange en face-à-face qui se déroule dans le temps immédiat. Un fil de discussion peut s'étaler sur quelques minutes comme en D1, fil constitué de deux messages publiés à 27 minutes d'intervalle. La valeur modale est de 16 jours, et une extrême variabilité est constatée au fil des mois (Figure 1). La durée moyenne sur l'ensemble de la session est de 4 jours et demi par discussion. Plusieurs discussions différentes peuvent avoir lieu simultanément, ce qui n'est pas possible en face-à-face. 


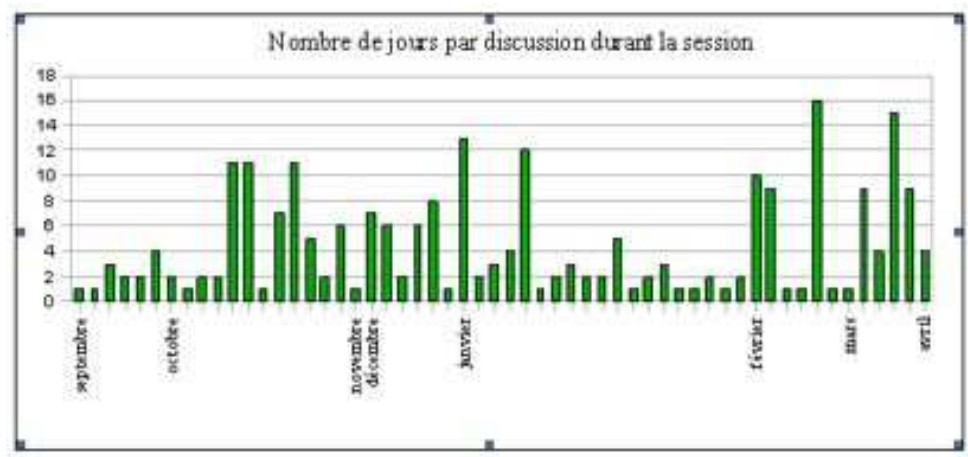

Figure 1 : Nombre de jours par discussion durant la session (Pradeau, 2009) p. 46.

Une discussion est constituée d'au moins deux messages. La valeur modale est de seize messages, avec une moyenne de six messages par fil sur la totalité de la session. Une présentation schématisée des échanges met en évidence une forme globalement linéaire des discussions (Figure 2). Les messages d'une discussion y sont représentés chronologiquement de gauche à droite. Par exemple, en D1, Denise répond à Ariane.

\begin{tabular}{|l|l|}
\hline$D 1$ & $A<A<D$ \\
\hline$D 4$ & $F<A<A<B \leq D \leq F$ \\
\hline$D 6$ & $C<F \leq D \leq A$ \\
\hline$D 10$ & $F<F<A \leq B<D<B<A<D<A<F$ \\
\hline$D 21$ & $B<B<D<A<A<F<A<D<A<A<B<D$ \\
\hline$D 42$ & $B E A<B$ \\
& $B E F<A<A$ \\
\hline
\end{tabular}

Figure 2 : Schématisation des fils de discussion (Pradeau, 2009) p. 48

Sur la session :

- Quatorze (14) discussions ne contiennent que deux messages sur le modèle de D1.

- Vingt-quatre (24) discussions, contenant au moins trois messages, sont strictement linéaires comme illustré en D6 ou D21.

- Dix-huit (18) autres sont non linéaires, comme D10 ou D42, sans toutefois se rapprocher d'un modèle circulaire ou plus complexe, que l'on pourrait retrouver dans une conversation en face-à-face. Cette linéarité contrainte par l'ossature technique et l'arborescence des échanges peut induire une forme de restriction à l'interactivité. En effet, un flou existe parfois dans la détermination de qui répond à qui, et les réponses peuvent être déposées davantage par commodité technique qu'en lien direct avec le contenu du message précédent. Il existe aussi des messages où l'étudiant se répond à lui-même comme c'est le cas en D21 pour Bernard et Ariane. L'observation des messages et de leur place dans le réseau apporte de nombreuses indications. La structure de chaque message est identique et donne différentes informations au lecteur : le numéro du message, son auteur, à quel message il répond, de quoi il traite, à quel moment il a été publié. La date et l'heure précise de son émission facilitent la reconstitution d'une chronologie des interactions. Le nom de l'auteur est cité après la mention "envoyé par", ce qui n'empêche pas chaque étudiant d'apposer presque systématiquement sa signature à la fin de son écrit. L'objet du message donne brièvement une indication générale sur le contenu du message. Cette information peut se diluer progressivement lorsqu'il existe de nombreuses réponses dont le contenu évolue au cours de l'échange.

Sur les trois cent trente-neuf messages qui constituent le corpus, cent dix contiennent une pièce jointe, soit presque un tiers. Ces pièces jointes sont essentiellement des fichiers issus de logiciels bureautiques et contenant des données en lien avec le travail collaboratif en cours. Grâce à l'échange de ces fichiers, les membres du groupe peuvent mutualiser leurs documents, découvertes, questionnements ou avancées du travail. Cette possibilité technologique n'est pas sans incidences sur le processus d'apprentissage en contribuant à l'échange rapide de connaissances. 
Une variation importante existe en ce qui concerne la taille des messages. Le plus court comprend un seul mot, "voilà", accompagné d'une pièce jointe. Le plus long comprend 626 mots, ce qui est considérable. Il s'agit d'un message déposé par Emile après une longue absence qu'il semble compenser par une participation plus forte concentrée sur un seul message, ce qui pourrait être assimilé à un échange réparateur. En face-à-face, cette action serait considérée comme une monopolisation de la parole, ce qui ne semble pas le cas ici, même si la règle d'usage dans le cadre d'un forum est plutôt à la concision.

\subsection{Aspects spatio-temporels}

La figure 3 permet de visualiser l'évolution des échanges sur l'ensemble de la session de travail collaboratif.

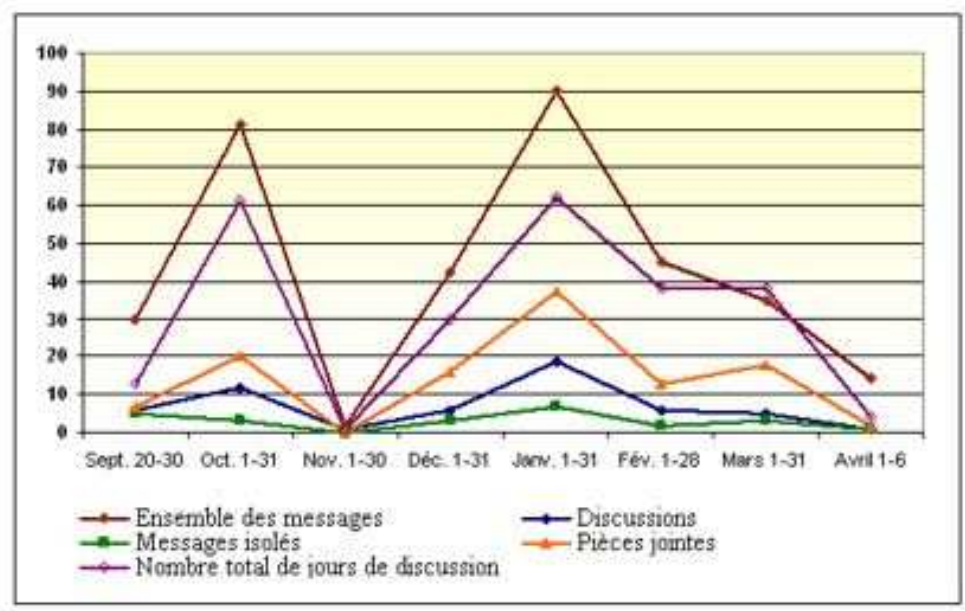

Figure 3 : Évolution des échanges au cours de la session de formation (Pradeau, 2009) p. 56.

Les périodes de mi-octobre et mi-janvier présentent des pics d'activités interactionnelles avec une plus grande quantité de messages échangés et des discussions qui s'étirent dans le temps. Une comparaison entre le nombre de discussions, leur durée, le nombre de messages publiés, le nombre de pièces jointes transmises, met en évidence une similitude des courbes représentatives. Il semble qu'il existe des phases où toutes les actions possibles sur le forum sont simultanément engagées.

L'observation des heures et dates de réponses aux messages montre qu'elles sont inégalement différées. L'écart entre la publication de deux messages qui génèrent une discussion peut aller de 2 minutes à presque 30 jours. Cette flexibilité du temps représente une particularité de la formation à distance et un atout exploité par les étudiants. Très souvent empêchés par des obligations professionnelles, ils utilisent cette souplesse dans la gestion de leur organisation. Dans les deux premières phases du travail collaboratif $(\mathrm{P} 1, \mathrm{P} 2)$, nous constatons que les unités de sens à connotations temporelles sont présentes dans un message sur deux. Ces références disparaissent en fin de session puisque l'objectif est atteint. Il serait intéressant d'étudier plus finement le rapport au temps pour chaque étudiant ainsi que l'incidence sur le processus d'apprentissage. En effet, en distanciel, l'apprenant est libre dans l'organisation de son temps de formation et doit donc planifier seul ses périodes de travail, ce qui nécessite une forte aptitude à l'autogestion.

Les messages font fréquemment référence à des notions d'espace. L'objet du message initiateur de la première discussion indique "C'est ici" comme pour tenter de définir un lieu de travail commun dans l'espace totalement immatériel du campus numérique. En D26M1433, Ariane confirme un rendez-vous sur un autre espace virtuel que celui fourni par FORSE : "20h30 demain donc sur la place du village virtuel ... msn dites oui!!:-) ". Ces références à des espaces extérieurs au forum étudié, à des lieux de clavardage ou rendez-vous téléphoniques (soit d'interactions en mode synchrone) sont conséquentes dans les deux premiers corpus (un message sur trois en P1, un message sur cinq en P2, aucun en P3). Le troisième corpus est moins significatif car il représente la phase finale de la session, où la multiplication des échanges semble moins nécessaire sur le plan du travail à effectuer. Cette diversité des lieux d'échanges laisse supposer que les interactions entre les étudiants ne sont pas limitées aux actions visibles sur le forum. D'autres régions existent, avec des zones qui sont davantage privées et où les modalités 
interactionnelles sont probablement différentes. Sur le forum, tous les messages ou documents mis en ligne sont visibles et consultables à tout moment par l'ensemble des étudiants de la promotion. Il est possible que cette large visibilité influence le contenu des messages et incite à la pratique d'échanges en dehors de cet espace, d'où la multiplication d'espaces d'interactions parallèles, comme déjà mis en évidence par une étude antérieure sur le campus FORSE (Béziat et Wallet, 2007) (p.70). Enfin, l'existence d'autres espaces sur le campus est rappelée par l'irruption de messages de personnes n'appartenant pas au groupe.

\subsection{Actions, présence, engagement}

Nous constatons un rapport de un à dix concernant le nombre de messages déposés sur le forum par les participants. Ariane en publie le maximum, soit 114, tandis que Carole est plus discrète avec seulement 11 messages publiés sur l'ensemble de la session.

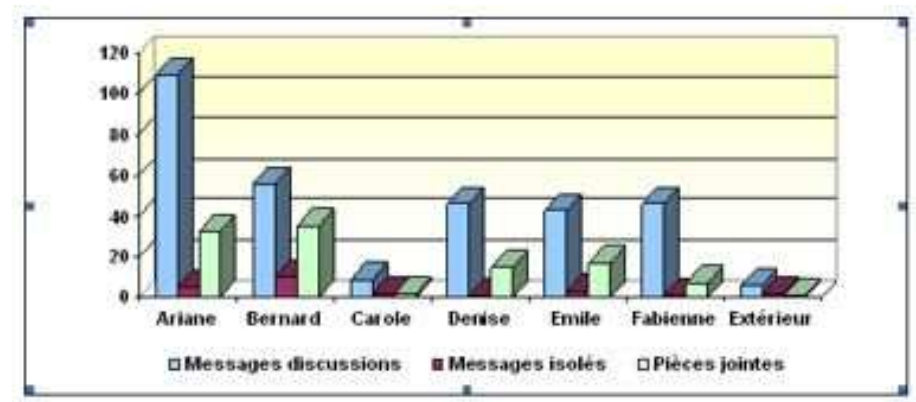

Figure 4 : Actions sur l'ensemble de la session (Pradeau, 2009) p. 60.

Ces différences d'implication se retrouvent tant dans la participation aux discussions que dans la transmission de pièces jointes. Le nombre d'actions de communication est donc variable d'un étudiant à l'autre mettant ainsi en évidence une forme d'engagement spécifique à chacun des interactants. Cette variabilité de l'engagement est déjà soulignée dans l'étude citée supra mettant en exergue une typologie des apprenants allant des "non participants" aux "hyperactifs" (Béziat et Wallet, 2007) (p.72).

Ariane est l'étudiante qui répond le plus souvent et le plus rapidement à un message initiateur, ce qui semble dénoter un engagement plus fort dans l'action. Elle marque plus massivement sa présence en multipliant les actions possibles dans le cadre de ce forum. A l'opposé, Carole est plutôt passive. Elle initie peu de discussions, répond peu aux messages initiateurs, transmet seulement deux pièces jointes, dépose son dernier message le 27 décembre et décroche du projet collaboratif à mi-parcours, modifiant ainsi la composition du groupe. Sa participation est proche de celle des intervenants extérieurs à l'équipe. Cette plus ou moins grande activité fait varier la visibilité de chacun et contribue probablement à modifier le sentiment de présence des uns et des autres dans le groupe. Une autre observation concerne la quantité de mots utilisés pour communiquer qui s'avère variable d'un étudiant à l'autre. Un comparatif entre Denise et Emile, dont l'engagement dans l'action est similaire (dépôts équivalents de messages ou pièces jointes), montre un écart important en ce qui concerne la taille moyenne des messages (en moyenne : Emile 93 mots par message ; Denise 40). Au-delà de ces aspects quantitatifs qui particularisent chaque étudiant, un constat intuitif en lecture flottante met en évidence des différences dans leur "façon d'être". Ce que chacun montre de lui le singularise. Suivant le langage qu'il privilégie, suivant son degré d'implication, chaque étudiant montre un fragment de son identité sociale, ou pour reprendre le concept d'Erving Goffman, une face bien spécifique.

\section{Apprentissage collaboratif à distance et concepts goffmaniens}

Les traces numérisées des actions des six étudiants donnent un nombre conséquent d'informations sur leurs interactions, ce qui souligne l'intérêt du courant théorique à visée microsociologique. Les résultats de l'observation déclinés supra confirment la richesse et l'aspect fortement structuré des interactions. Ce contexte particulier d'enseignement-apprentissage contraint les acteurs mais leur offre paradoxalement un vaste champ de liberté. Leurs actions individuelles agissent sur l'organisation sociale qui agit à son tour sur chacun des interactants. Nous allons dans cette dernière partie nous attacher à développer l'aspect 
inférentiel de cette étude en examinant les concepts proposés par Erving Goffman pour considérer leur congruence aux interactions sociales sans coprésence physique.

\subsection{Forum et cadre de l'expérience}

Goffman émet l'hypothèse qu'en s'intéressant à une situation ordinaire on se pose la question : "Que se passe-t-il ici ?" (Goffman, 1974b) (p.16). L'expérience sociale des échanges sur un forum de discussion représente-t-elle une "situation ordinaire" ? Sur un campus numérique, cette forme d'organisation des interactions est effectivement usuelle tant les forums et espaces d'échanges asynchrones y sont systématiquement proposés. La multiplication actuelle des réseaux sociaux laisse supposer que ces modalités interactionnelles à distance vont peu à peu devenir des situations ordinaires, et ce malgré les fortes disparités existant encore en terme d'infrastructures et d'alphabétisation numérique. Un forum peut être assimilé à un cadre particulier où les acteurs sont engagés dans l'interaction avec un objectif déterminé. Sur le forum étudié, les étudiants ont un travail à réaliser en commun, ce qui les rend interdépendants. En observant leurs traces numériques, nous avons tenté de répondre à la question : que se passe-t-il sur un forum de discussion proposé sur un campus numérique dans un objectif d'enseignement et d'apprentissage ? Nos observations ont montré sous quelles formes et avec quelles contraintes s'organisent les échanges. La structure du forum agit sur les interactants, qui eux-mêmes déterminent l'expérience sociale par les actions réalisées. Ce cadre structuré, fortement imprégné d'aspects technologiques, influence les individus et leurs perceptions. Les impératifs académiques ajoutent des éléments astreignants qui balisent cette expérience sociale. Nous faisons référence au quotidien à une multitude de cadres s'imbriquant les uns dans les autres. Une salle de classe est un cadre de l'expérience, un forum de discussion en est un autre. L'un existe dans un espace physiquement délimité, l'autre n'est pas aussi précisément situé, ce qui en fait cependant un cadre particulier de l'expérience, où les acteurs sociaux interagissent. La capacité d'un étudiant à maîtriser les outils et usages du web peut largement conditionner ses actions sur le forum, en facilitant ou limitant ses possibilités de communiquer avec les autres membres du groupe. L'engagement interactionnel de chacun est tributaire de ce contexte particulier. Nous avons constaté combien sont structurées et limitées les actions possibles pour les étudiants, au point qu'ils en développent de nouvelles en dehors du campus élargissant ainsi cette expérience sociale. Nous avons montré combien les discussions et les messages présentent une architecture particulière, plutôt linéaire pour les discussions et sur un modèle épistolaire pour les messages. Ces différentes remarques nous conduisent à proposer de considérer un forum de discussion comme un cadre de l'expérience avec ses règles explicites et implicites perçues sur un mode plus ou moins consensuel par les participants à l'interaction.

\subsection{Forum, scène, coulisse}

L'analyse du contenu des messages a mis en évidence le nombre conséquent de références à des espaces autres que le forum dédié. Différents lieux d'interactions sont proposés par l'université en début de session. Ils sont ensuite multipliés en cours de module à l'initiative de certains étudiants. Pour reprendre les notions de régions chères à Goffman, nous proposons d'assimiler l'espace du forum de discussion proposé aux six étudiants à ce qu'il a nommé la scène. Selon sa métaphore théâtrale, c'est dans cette région que se déroule la représentation et où chacun des étudiants préserve sa face et celle des autres. Le public de cette représentation rassemble les enseignants et les autres étudiants de la promotion qui peuvent techniquement voir ces échanges mais aussi déposer des messages, comme en D19M940 où Geneviève, qui ne fait pas partie du groupe d'étudiants, écrit : "bonjour, je me permets de m'immiscer ... pour vous proposer cette lecture qui peut-être vous intéressera.... bon courage pour la suite! http://www.inrp.fr Geneviève". Notons l'utilisation du mot "s'immiscer" qui semble indiquer une forme d'intrusion dans un espace non prévu à cet effet, alors qu'aucune règle précise n'a été énoncée dans ce sens. Les actions implicitement acceptées sont donc fonction du lieu où elles se réalisent. Les lieux de clavardage hors campus, souvent évoqués dans les messages, ou encore les échanges par courriels ou contacts téléphoniques se rapprochent de ce que Goffman a appelé les coulisses. Dans ces espaces privés, les étudiants du groupe de travail collaboratif peuvent davantage se laisser aller, car ils ne sont plus en scène sous le regard du public. Ce qui se joue dans ces coulisses serait à explorer car probablement déterminant pour l'ensemble de l'interaction. La région extérieure est représentée par tous les autres espaces du campus numérique, ceux où interviennent les enseignants et les autres étudiants, mais aussi les personnels administratifs et techniques, avec lesquels les étudiants peuvent aussi avoir des échanges. Sur les trois cent trente-neuf messages du forum, huit sont déposés par trois étudiants qui ne font pas partie du 
groupe, et semblent ainsi provenir de cette région extérieure.

L'enregistrement des messages du forum et l'archivage permettent de revoir la scène de l'interaction à tout moment. Cette particularité, ainsi que l'aspect public des échanges ont-ils des effets sur la communication ? Existe-t-il une forme de mise en scène plus ou moins consciente de la part de chacun des acteurs? Enfin, quelle est son incidence sur le travail collaboratif et son évaluation ? La visibilité plus ou moins importante des étudiants influence-t-elle les enseignants ou tuteurs ? De nombreuses questions sont induites par l'exploration de ce forum.

\subsection{Forum et ordre de l'interaction}

La question fondamentale de l'œuvre de Goffman porte sur ce qu'il a nommé l'ordre de l'interaction, c'est-à-dire ce qui s'organise explicitement et implicitement lors des échanges. Comment rendre opératoire ce concept pour lire et comprendre une rencontre sans face-à-face sur un forum de discussion? Plusieurs éléments peuvent être évoqués. La scène, représentée par le forum, est l'espace où un certain nombre de personnes semblent "habilitées" à agir, soit dans le cas présent les six étudiants du groupe de travail collaboratif. Le participant ratifié est celui qui est à sa place dans l'ordre de l'interaction (Joseph, 1998) (p.66). Cette règle implicite, ce droit de se déplacer dans certaines régions, fait partie d'un ordre interactionnel qui, lorsqu'il est profané, déclenche des échanges réparateurs. La discussion D41 illustre ce propos : Geneviève, étudiante extérieure au groupe, y dépose deux messages. Le premier correspond à ce que Goffman a appelé une rupture de cadrage: Geneviève poste un message accompagné d'une pièce jointe destinée aux étudiants de son groupe, alors qu'elle aurait dû le déposer sur un autre forum. Le deuxième ressemble à un échange réparateur sous forme d'une excuse concernant son erreur. Sur un registre similaire, en I10, Geneviève agit selon ses propos de façon osée tout en demandant leur indulgence à l'ensemble des étudiants du groupe : "Objet : récup. bonsoir.. je me suis permis de récupérer un travail de Bernard.....et comme il est déjà tout prêt chez vous et sans doute mieux que ce que j'aurais pu faire.......voilà ! oui, c'est gonflé, j'espère néanmoins que vous ne m'en voudrez pas trop! /.../! à bientôt". Soulignons l'utilisation de la formule "chez vous" qui participe à la délimitation de territoires évoquée plus haut. En face-à-face, cette action aurait été probablement précédée d'une demande de partage formulée auprès de Bernard. Dans le cas présent, cette action n'amène aucune réaction visible, le message reste isolé. L'ordre interactionnel est modifié sur un forum, dont le potentiel de mutualisation semble implicitement accepté par l'ensemble des participants.

L'aspect public des échanges fait partie de l'ordre interactionnel. Ainsi, les réponses à un message ne sont pas forcément adressées à son émetteur. Chacun semble répondre à l'ensemble du groupe, et il n'existe pas de réelle cohérence dans la chaîne des réponses du moins quant aux destinataires des messages. C'est probablement ce qui provoque la forme très linéaire des fils de discussion, avec à l'extrême des réponses à soi-même que l'on ne trouve pas en face-à-face. Enfin, le fait qu'une réponse soit différée de plusieurs jours ne s'avère pas être une fausse note dans le jeu interactionnel, mais semble faire partie de l'ordre des choses.

\subsection{Equipe, sens commun, engagement}

Le concept d'équipe développé par Goffman dans La mise en scène de la vie quotidienne - La présentation de soi (1973a) nous semble applicable aux groupes inter-agissant à distance. Dans le cadre d'un travail collaboratif en ligne, les étudiants sont engagés dans la réalisation d'un projet commun puisqu'il est question d'une production collective avec une échéance précise. De plus, dans le cas étudié, il existe un enjeu fort puisqu'une partie du travail collaboratif conduit à des productions intervenant dans l'évaluation finale. De là nous pouvons considérer qu'il doit exister une relation d'interdépendance entre les membres du groupe. Chaque équipier compte sur le respect de l'ordre de l'interaction par ses partenaires. Ils sont reliés par un sens commun donné à la situation en cours et une forme de familiarité s'instaure dans l'équipe. Un minimum de participation est implicitement reconnu par tous comme indispensable, au point que les défaillances passagères des participants créent des fausses notes dans l'ordre interactionnel et déclenchent des rappels à l'ordre ou des échanges réparateurs. Ce non respect de la représentation est souligné en D26M1399, où Bernard apostrophe certains membres du groupe qui sont dans une phase de moindre implication : "Denise, Ariane ... et les autres! Denise, Ariane ... Je vois que vous avez bien bossé pdt mes quelques jours d'absence /.../ je me un peu demande comment nos collègues (momentanément furtifs) vont pouvoir se raccrocher/.../BIEN SûR, ces remarques non pas pour casser ce 
qui a été fait /.../ mais pour jouer le jeu du "collaboratif". J'espère maintenant que Emile, Fabienne et Carole vont pouvoir réagir, .../". Notons au passage l'utilisation de l'expression "jouer le jeu" qui nous ramène à la métaphore théâtrale. De même, en D25M1393, Carole, qui est perçue comme trop passive, se fait interpeller par Ariane sur un mode humoristique : ".../ pour la méthodo, tu dis que tu vas essayer de "suivre" mais cocotte, on a aussi besoin de forces vives !!!! en plus t'es bonne en stat. alors ca te dit pas de faire le tableau des variables? c'est une proposition parmi d'autres ;-)". Carole ne donnera pas suite à cette injonction qui arrive en réponse à son message d'appel à l'aide qui sera aussi son dernier message posté pour cette session (dont l'objet était "Vraiment marre..." et dont le contenu débutait par "Au secours" pour aborder ensuite des difficultés essentiellement d'ordre technique). Emile illustre aussi cette interprétation en D23M1385 où il s'excuse pour une nouvelle absence à un clavardage. Plus loin, il dépose un long message réparateur pour justifier son manque de participation tout en s'excusant pour ses futures absences. Cet étudiant sera le plus actif dans l'interaction en fin de session comme pour compenser ses difficultés dans l'engagement en début et au cours du module.

Dans une situation distancielle, il semble qu'une convention sous-jacente entre les acteurs postule que chacun marque sa présence par les actes qu'il pose, cette pro-activité étant une condition de l'interaction. Un trop grand écart dans la mobilisation de chacun des participants engagés dans un travail collaboratif peut-il nuire à la qualité interactionnelle au sein du groupe ainsi qu'aux processus d'enseignement et d'apprentissage ? Un long silence peut-il rester sans conséquences ? N'y a-t-il pas une forme d'ordre de l'interaction ou une obligation sociale implicite de réaliser un certain nombre d'actions visibles pour rester impliqué dans le groupe et comment remédier au risque d'exclusion virtuelle?

\subsection{Face et distance}

Nous avons repris les analyses de (Blandin, 2004) mentionnant un continuum s'étalant entre deux pôles déterminés par la notion de présence et celle d'absence. En nous appuyant sur ce gradient pour modéliser le sentiment de présence, il nous semble que le concept de face peut tout à fait être opératoire pour analyser et interpréter les échanges produits sur un forum. Chacun des acteurs montre une part de lui-même par les actions qu'il engage et la façon dont il communique. Cette part de soi qui s'expose à distance passe essentiellement par l'écrit, contrairement à ce qui se passe en présentiel. "L'identité individuelle est circonstancielle et faite de différentes facettes" (Le Breton, 2004) (p.139). Ce qui se donne à voir, en présence ou à distance, serait en quelque sorte l'une de ces multiples facettes. L'observation des traces d'échanges sur le forum laisse apparaître des disparités concernant l'engagement dans l'action. Emile semble avoir des difficultés à gérer de front ses obligations professionnelles et universitaires. Il fait souvent référence à ses difficultés, ce qui déclenche le soutien et les encouragements des autres membres du groupe. Malgré l'expression fréquente de ses difficultés, il est le plus actif en fin de session et c'est lui qui dépose les documents finaux réalisés par le groupe. Il conclue la session par une dernière intervention de même qu'il avait participé à son ouverture en déposant le premier message isolé. Ariane montre une face très différente dans ce contexte spécifique. Elle multiplie les actions de communication et prend une place importante dans le groupe. Elle représente le participant de l'équipe qui semble diriger et contrôler la progression de l'action dramatique (Goffman, 1973a) (p.96). Ces disparités entre ce que chacun montre de lui tout en se conformant à des impératifs contextuels posent d'autres questions. Par exemple, existe-t-il une analogie entre ce qu'un acteur montre de lui-même en présentiel ou à distance ? Ou encore quelles sont les représentations générées chez les autres étudiants par la face exposée par chacun? Des études complémentaires seraient nécessaires pour tenter de répondre à ces interrogations.

\subsection{Rites interactionnels à distance}

Goffman soutient que les interactions sociales sont régies par des codes ou des rituels qui déterminent les comportements individuels (Goffman, 1974a). Dans le cadre du forum, nous avons observé des éléments rituels : les messages sont introduits et conclus de façon quasi-systématique par des salutations, dont certaines ont une connotation affective. Presque tous les messages se terminent par une signature, malgré l'indication en en-tête du nom de l'auteur. Sans concertation préalable, les six étudiants organisent leurs messages avec ces formes de parenthèses rituelles (Blandin, 2004) (p.372). Ces pratiques sont d'ailleurs beaucoup plus générales et habituelles sur Internet, où elles se retrouvent dans les messages électroniques. Le contenu écrit induit probablement ces similitudes avec des codes épistolaires. Pour les discussions, nous n'observons pas de pratiques sociales rituelles comme c'est le cas dans une conversation en 
face-à-face avec des salutations ou comportements annonciateurs de début ou de fin de l'échange. Leur structure linéaire, leur durée, la dispersion géographique des participants, le fait que plusieurs discussions peuvent se dérouler sur une même période, en font une forme très particulière d'interaction sociale. Cette organisation interactionnelle singulière se structure dans un espace non clairement situé physiquement et avec un rapport au temps modifié. Une observation d'un autre forum à vocation différente pourrait contredire ce constat. En effet, sur les forums thématiques, l'usage veut que chaque fil traite d'un sujet précis, et qu'il soit conclu, une fois le thème épuisé. Ces codes ne semblent pas encore suffisamment assimilés par les étudiants pour être d'un usage courant en campus numérique. La complexité de la communication à distance est renforcée par l'apparition de nouveaux codes de langage, tels que les émoticones ou les acronymes comme LOL ou MDR pour signifier des éclats de rire. L'ordre cérémoniel semble ainsi régi par la capacité des acteurs à partager des codes et représentations liés au contexte social particulier des interactions à distance. Il est possible que la banalisation de l'usage des artefacts techniques ainsi que la multiplication des interactions à distance contribuent peu à peu à une intégration de cette étiquette numérique. Il existe d'ailleurs sur le Web une Netiquette (contraction de Net et Etiquette). Cette charte, en définissant des règles de conduites sur Internet, participe à l'instauration progressive de ces nouvelles règles interactionnelles.

\section{Conclusion}

Cette approche microsociologique menée à un niveau qui se situe entre le microscopique et le mésoscopique, permet de prêter une attention particulière à ce qui se joue au niveau d'une petite unité sociale dans le contexte spécifique de l'apprentissage collaboratif en ligne. L'étude des dimensions sociales dans les situations d'enseignement-apprentissage instrumentées est à développer tant elles imprègnent tout processus de formation. Approfondir ces connaissances pourrait contribuer à l'amélioration des dispositifs et peut-être limiter le nombre d'étudiants quittant la scène prématurément.

Comme toute recherche en sciences humaines et sociales, cette étude reste cependant partielle et relative au point de vue adopté. Comme interroge Van Der Maren, "La loupe déforme t-elle la perception ?" (Van Der Maren, 1995) (p.113), question récurrente dans les débats tant méthodologiques qu'épistémologiques. A quel degré de représentativité et de fidélité, les données recueillies ou plutôt construites sont-elles le reflet des interactions au travers des traces manifestes laissées par des échanges? Nous avons tenté d'analyser minutieusement ces traces d'interactions, en tentant d'expliciter au mieux les critères mis en œuvre pour construire nos interprétations. "Si le sens se construit dans l'interaction, étant en quelque sorte un produit de cette interaction, avec un large spectre de valeurs possibles en cours d'échange, aucune grille ne pourra capter complètement ce qui se passe pendant le déroulement du forum. On ne peut que reconstruire des histoires a posteriori. Mais les informations que l'on peut fournir en cours, donnant une interaction enrichie, participent de cette évolution du sens" (Bruillard, 2005).

A la fois riches et hétérogènes, les recherches sur les forums de discussion présentent un caractère complexe (Henri et al., 2007). Pour extraire du sens des données, nous avons fait le choix, parmi d'autres possibles, d'un cadre de référence à versant interactionniste. Les concepts de face, de cadre de l'expérience, d'équipe, d'engagement, de scène et de coulisses, examinés dans ce contexte d'échanges sans coprésence physique, nous semblent congruents à ce contexte particulier. Les actions des apprenants sont contraintes par l'ossature technique du forum, mais aussi par une forme d'ordre interactionnel. Les étudiants paraissent guidés par des règles implicites qui s'apparentent à des pratiques rituelles. Leurs actes et ce qu'ils montrent d'eux-mêmes dans leurs écrits ne sont pas neutres dans le déroulement de l'action et du processus d'apprentissage. Ils ajoutent spontanément des espaces d'échanges à ceux proposés par l'université agissant ainsi sur l'organisation sociale. La réalité sociale du groupe de travail collaboratif observé est en permanence remodelée par les actions de ses membres. Nous constatons la disparition à mi-parcours d'un des étudiants de l'équipe, la plus forte implication de l'un d'entre eux, des variations dans l'engagement pour d'autres, des différences entre ce que chacun montre de lui-même.

Les notions de flexibilité de temps et de déterritorialisation de l'espace, largement évoquées dans la littérature sur la formation en ligne, se retrouvent dans cette approche micrologique. Nous constatons par ailleurs une répartition des espaces virtuels d'interactions en fonction du contenu de l'action dramatique, soit ici la réalisation d'un travail collaboratif en ligne. Ces éléments participent à la création de ce cadre de l'expérience spécifique que représente un forum à vocation éducative. Goffman détermine une région 
d'interaction comme "tout lieu borné par des obstacles à la perception, ceux-ci pouvant être de différentes natures" (Goffman, 1973a), p.105. Cette question de la délimitation de régions d'interactions ouvre à plus grande échelle une autre problématique. En effet, à un niveau macrosociologique, l'impossibilité pour une part importante de la population d'accéder au Web pose la question d'une démocratisation de l'accès à ces nouveaux espaces de formation, à cette nouvelle scène de représentation. Comme nous l'avons signalé, nous poursuivons nos travaux sur les effets identifiables des interactions produites au sein des situations organisées par une telle ingénierie pédagogique sur le développement conceptuel en statistique des sujets.

\section{Références}

ARINO M. (2001). L'écran comme nouveau territoire des relations sociales, Esprit critique, vol.03 no.10. Disponible en ligne : http://www.espritcritique.fr/0310/article7.html (consulté le 19/08/08)

AUDRAN J., (2006). La prise de parole sur les forums : un moyen d'évaluer les conditions de réussite d'un enseignement "en ligne" ? Revue Eduquer n¹2, pp.23-41. Disponible en ligne : http://www.audran.net/articles/index.htm (Consulté le 26/12/08)

AVEROUZ M., TOUZOT G., (sd), (2002) Rapport "Campus numériques, enjeux et perspectives pour la Formation Ouverte et à Distance", À l'attention de ministère de l'éducation nationale, du ministère de la recherche, de la Délégation à l'aménagement du territoire et à l'action régionale. http://lesrapports.ladocumentationfrancaise.fr/BRP/024000223/0000.pdf (Consulté le 15 janvier 2009)

BARDIN L. (2007). L'analyse de contenu. Paris, Quadrige PUF

BEZIAT J., WALLET J. (2007). Entre dispositif de formation et pratiques sociales : l'étudiant à distance, Dans Le campus numérique FORSE, analyses et témoignages, Publication des universités de Rouen et du Havre

BLANCHET A., GHIGLIONE R., MASSONNAT J., TROGNON A., (2000). Les techniques d'enquête en sciences sociales, Dunot, Paris

BONICCO C. (2007). Goffman et l'ordre de l'interaction, Un exemple de sociologie compréhensive, Philosorbonne $\mathrm{n}^{\circ} 1 /$ Année 2006-07, p 31-48, Disponible en ligne : http://edph.univ-paris1.fr/phs1/phs1.html (consulté le 13 février 2009)

BRUILLARD E. (2005). Le forum de discussion, un cas d'école pour les recherches en EIAH, Revue STICEF Sciences et Technologies de l'Information et de la Communication pour l'Éducation et la Formation. Editorial Volume 15. Disponible en ligne : http://sticef.univ-lemans.fr/num/vol2006/sticef 2006 ns edito.htm (consulté le 2 février 2010)

BRUILLARD E. (2008). Travail et apprentissage collaboratifs dans des formations universitaires de type hybride. Éléments de réflexion. Revue Éducation - Formation - e-288, Université de Mons-Hainaut. En ligne http://ute2.umh.ac.be/revues /index.php?revue $=4 \&$ page $=3$

DILLENBOURG P. (1999). What do you mean by collaborative learning? In P. Dillenbourg (Ed) Collaborative-learning: Cognitive and Computational Approaches, Oxford, Elsevier, p.1-19. Disponible en ligne : $\underline{\mathrm{http} / / \text { tecfa.unige.ch/tecfa/publicat }}$ /dil-papers-2/Dil.7.1.14.pdf (Consulté le 15/11/08)

FICHEZ E. (2006). Campus numériques français, peut-on parler de réussite ou d'échec ? Informations, Savoirs, Décisions, Médiations, Journal International des Sciences de l'Information et de la Communication, ISDM N²5. Disponible en ligne : http://isdm.univ-tln.fr/PDF/isdm25/Fichez TICE206.pdf (Consulté le 17/01/09)

FORSE. FOrmation et Ressources en Sciences de l'Education : http://www.sciencedu.org/

GLIKMAN V. (2002). Des cours par correspondance au "E-learning", Education et formation, Paris, Presses Universitaires de France.

GOFFMAN E. (1973a). La mise en scène de la vie quotidienne, 1-La présentation de soi, Le sens commun, Paris, Les Éditions de Minuit

GOFFMAN E. (1973b). La mise en scène de la vie quotidienne, 2-Les relations en public, Le sens commun, Paris, Les Éditions de Minuit

GOFFMAN E. (1974a). Les rites d'interactions, Le sens commun, Paris, Les Éditions de Minuit

GOFFMAN E. (1974b). Les cadres de l'expérience, Traduction (1991) I. Joseph, M. Dartevelle, P. Joseph, Paris, Les Éditions de Minuit

GOFFMAN E. (1988). Les Moments et leurs Hommes, textes réunis et présentés par Yves Winkin, Paris, Seuil/Minuit.

HENRI F., PERAYA D., CHARLIER B. (2007). La recherche sur les forums de discussion en milieu éducatif : critères de qualité et qualité des pratiques. Revue STICEF Sciences et Technologies de l'Information et de la Communication pour l'Éducation et la Formation. Disponible en ligne : http://sticef.univ-lemans.fr/num/vol2007/18-henri/sticef 2007 henri 18p.pdf (consulté le 08/08/08) 
JACQUINOT-DELAUNAY G. (2001). Le "sentiment de présence". Actes des Deuxièmes Rencontres Réseaux Humains / Réseaux Technologiques. Poitiers, 24 juin 2000. Documents, Actes et Rapports pour l'Education, CNDP, p. 183-191. Disponible en ligne : http://edel.univ-poitiers.fr/rhrt/document.php?id=773 (consulté le 25/08/2008)

JOSEPH I. (1998). Erving Goffman et la microsociologie, Paris, Philosophie PUF.

LE BRETON D. (2004). L'interactionnisme symbolique, Paris, Presses Universitaires de France.

LEVY P. (1997). Cyberculture. Rapport au conseil de l'Europe, Paris, Editions Odile Jacob

MANGENOT F., (2008). Pratiques pédagogiques instrumentées et propriétés des outils : le cas des forums. Revue STICEF Sciences et Technologies de l'Information et de la Communication pour l'Éducation et la Formation. Volume 15. Disponible en ligne : http://sticef.univ-lemans.fr/num/vol2008/05-mangenot/sticef 2008 mangenot 05.htm\# (Consulté le 28/02/09)

PEIRCE C.S, (2006). Cuvre philosophiques - Tome 3, Ecrits logiques. (Trad. C. Engel-Tiercellin et P. Thibaud, (org)) Paris: Cerf PERRET-CLERMONT A.-N, (1986). La construction de l'intelligence dans l'interaction sociale, Berne : Peter Lang, 3ème éd., (1ère éd. 1979)

PRADEAU A. (2009). Des interactions sociales en formation universitaire à distance. Une approche microsociologique exploratoire et inférentielle. Mémoire de Master1 en Sciences de l'Éducation Université de Lyon-Lyon2 (dirigé par Jean-Claude Régnier)

QUEAU P. (2000). La planète des esprits, Pour une politique du cyberespace, Paris, Éditions Odile Jacob.

ROSCH E. H. (1975) Cognitive representations of semantic categories, Journal of Experimental Psychology, 104, 192-233

SIMEONE A., ENEAU J., RINCK F., (2007). Scénario d'apprentissage collaboratif à distance et en ligne : des compétences relationnelles sollicitées ou développées? International Journal of Information Sciences for Decision Marketing n 29. Disponible en ligne : http://isdm.univ-tln.fr/articles/num archives.htm\#isdm29 (Consulté le 21 novembre 2008).

THOLOZAN O. (2004). Temporalité et contenu communicationnel d'un enseignement en ligne : l'exemple du DAEU à la MFC de l'Université d'Aix-Marseille III , Informations, Savoirs, Décisions, Médiations, Journal International des Sciences de l'Information et de la Communication, ISDM N${ }^{\circ}$. Disponible en ligne : $\underline{\text { http://isdm.univ-tln.fr/PDF/isdm18/58-tholozan.pdf }}$ (Consulté le 14/01/09)

VAN DER MAREN J-M. (1995). Méthodes de recherche pour l'éducation, Montréal, Presses de l’Université de Montréal., De Boeck Université.

WALLET J. (2002). Le point de vue des sciences de l'éducation, Actes du symposium Technologies informatiques en éducation : perspectives de recherches, problématiques et questions vives, Paris MSH/INRP, 2002.

WALLET J., (2004). Entre pratiques réflexives et approches théoriques en formation à distance, questions croisées, Distances et savoirs 2004/1, Volume 2, p. 9-23.

WALLET J. (2007). Le campus numérique FORSE, analyses et témoignages, Publication des universités de Rouen et du Havre, Textes réunis par $\mathrm{J}$. Wallet

WEISSBERG J.-L. (2001). Entre présence et absence. Actes des Deuxièmes Rencontres Réseaux Humains / Réseaux Technologiques. Poitiers, 24 juin 2000. Documents, Actes et Rapports pour l'Education, CNDP, p. 31-39. Disponible en ligne : http://edel.univ-poitiers.fr/rhrt/document.php?id=429 (consulté le 29/07/2008)

WINKIN Y. (2001). Anthropologie de la communication, De la théorie au terrain, Paris, Éditions De Boeck \& Larcier S.A. / Editions du Seuil.

WOLTON D., (2000). Internet et après, Une théorie critique des nouveaux médias, Paris, Flammarion

\section{ANNEXES}

\section{Fiche-Guide}

Année 2006-2007 - Université Lumière Lyon2 -Master1 SPEF

Dispositif FOAD Campus Numérique FORSE

Cours de méthodes quantitatives et qualitatives de J-C. Régnier et F. Clerget

\section{Démarche générale :}

L'objectif du cours est double :

For compléter la formation méthodologique acquise en licence en particulier dans le domaine des 
méthodes quantitatives [A], méthodes qualitatives [B] et de la statistique.

FOM aider à mettre en lien les acquis méthodologiques dans le cadre de la recherche conduite pour le mémoire de master1 (maîtrise)

RÉSUMÉ : Pour atteindre cet objectif, une situation problème est proposée autour d'un thème global commun à tout le groupe, prétexte à la mise en œuvre des outils et méthodes visées dans la formation. Ce thème est celui de l'article [Doc8] et [Doc9] que nous avons étudié lors de la séance présentielle à l'université Lyon 2 le 7 septembre 2006 (9h-10h30).

\section{Thème : La réussite scolaire}

\section{Mode d'organisation du travail collaboratif :}

RÉSUMÉ : Il s'agit de constituer des groupes d'au plus 6 étudiants) autour des sous-thèmes :

\begin{tabular}{|l|l||l|l|}
\hline Sous-thème 1 & Sous-thème 2 & Sous-thème 3 & Sous-thème 4 \\
\hline $\begin{array}{l}\text { Réussite } \\
\text { scolaire et } \\
\text { climat familial }\end{array}$ & $\begin{array}{l}\text { Réussite } \\
\text { scolaire et } \\
\text { genre }\end{array}$ & $\begin{array}{l}\text { Réussite } \\
\text { scolaire et } \\
\text { travail en } \\
\text { équipe } \\
\text { pédagogique }\end{array}$ & $\begin{array}{l}\text { Réussite } \\
\text { scolaire et } \\
\text { classes } \\
\text { sociales }\end{array}$ \\
\hline
\end{tabular}

\section{Tâches :}

\section{Phase 1 : Construire des données}

- RÉSUMÉ : Cette tâche est à réaliser en groupe et le produit final est celui du groupe. Chaque groupe devra construire un questionnaire [Prod1A] et un guide d'entretien [Prod1B] dans le but de produire des données pertinentes relatives à une investigation du sous-thème. Le questionnaire devra comporter entre 8 à 10 questions couvrant l'ensemble des formats possibles (voir [DOC6]). Le guide d'entretien: en référence au cours, choisir un type d'entretien ; argumenter ce choix en lien avec l'avancée supposée de la recherche en cours (phase exploratoire, phase de vérification). Définir et argumenter le choix de la population parente ; construire le guide d'entretien qui comportera à la fois les items et les points à aborder. [Prod2A] [Prod2B] Les protocoles de mise au point et de passation du questionnaire devront être explicités.[Prod3Aa] Un tableau sera réalisé sous Excel permettant de recevoir les réponses aux questions lors du dépouillement des questionnaires. [Prod3Ab] Compléter le tableau [Prod3Aa] par des données réellement construites ou par des données simulées.[Prod3Ba] Construire le protocole de dépouillement des entretiens. [Prod3Bb] Alimenter l'outil ainsi construit par des informations réellement recueillies ou par des informations simulées.

\section{Phase 2 : Traiter des données (projet)}

Cette tâche est à réaliser en groupe et le produit final est individuel. [Prod4A/B] : à partir du [Doc9], en recourant à une approche quantitative et à une approche qualitative, analyser les informations contenues dans le tableau en les mettant en relation avec le titre, le résumé de l'article, et le commentaire du tableau. Rendre compte individuellement de cette analyse en deux pages maximum. Il s'agira en particulier de bien préciser le test statistique auquel renvoie ce tableau.

Cette tâche est à réaliser en groupe et le produit final est celui du groupe

- RÉSUMÉ : À partir du questionnaire, prévoir plusieurs traitements des données qui mettent en œuvre la description statistique et l'inférence statistique. La mise en œuvre d'un test d'hypothèses concernant le croisement de deux questions (de type variables qualitatives) figure parmi les traitements attendus. [Prod5A] Ce protocole de traitement sera explicité et rédigé clairement en deux pages maximum. À partir du guide d'entretien, prévoir [Prod5B] le croisement et l'ordonnancement des informations recueillies.

\section{Phase 3 : Traiter des données (effectuation)}

Cette tâche est à réaliser en groupe et le produit final est celui du groupe 
RÉSUMÉ : [Prod6A/B] Il s'agira de mettre en œuvre les traitements annoncés dans [Prod5A] à partir des tableaux des séries statistiques [Prod3 Ab] et [Prod5B] à partir de [Prod3Bb]. Ces traitements seront rédigés proprement à la manière d'une communication d'une étude sous la forme d'un document qui peut ressembler à un article, à une affiche sous Powerpoint.

\section{IV. échéances :}

\begin{tabular}{|c|c|c|c|}
\hline $\begin{array}{l}\text { Produit } \\
\text { attendu }\end{array}$ & Modalité & $\begin{array}{l}\text { Date de } \\
\text { remise } \\
2007\end{array}$ & Contenu produit \\
\hline$\left[\begin{array}{l}{[\operatorname{Prod} 1 \mathrm{~A}]} \\
{[\text { Prod1B }]}\end{array}\right.$ & $\begin{array}{l}\text { Un par } \\
\text { groupe }\end{array}$ & $\begin{array}{l}31 \\
\text { janvier }\end{array}$ & \begin{tabular}{|l} 
Questionnaire (Word) \\
Guide d'entretien (Word)
\end{tabular} \\
\hline$\left[\begin{array}{l}{[\operatorname{Prod} 2 \mathrm{~A}]} \\
{[\operatorname{Prod} 2 \mathrm{~B}]}\end{array}\right.$ & $\begin{array}{l}\text { Un par } \\
\text { groupe }\end{array}$ & $\begin{array}{l}31 \\
\text { janvier }\end{array}$ & 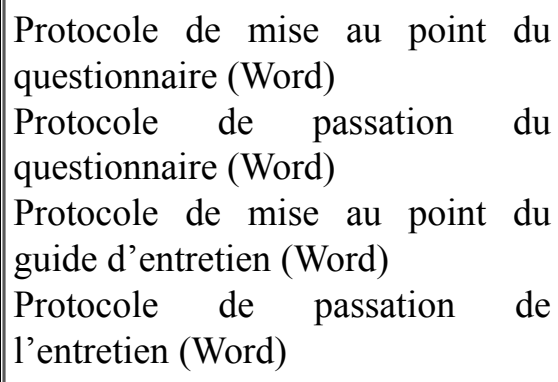 \\
\hline $\begin{array}{l}{[\operatorname{Prod} 3 \mathrm{Aa}]} \\
{[\operatorname{Prod} 3 \mathrm{Ba}]}\end{array}$ & $\begin{array}{l}\text { Un par } \\
\text { groupe }\end{array}$ & $\begin{array}{l}31 \\
\text { janvier }\end{array}$ & $\begin{array}{l}\text { Tableau des séries statistiques } \\
\text { avant dépouillement (Excel) } \\
\text { Protocole de dépouillement des } \\
\text { entretiens }\end{array}$ \\
\hline$\left[\begin{array}{l}{[\operatorname{Prod} 3 \mathrm{Ab}]} \\
{[\operatorname{Prod} 3 \mathrm{Bb}]}\end{array}\right.$ & $\begin{array}{l}\text { Un par } \\
\text { groupe }\end{array}$ & $\begin{array}{l}11 \\
\text { février }\end{array}$ & $\begin{array}{l}\text { Tableau des séries statistiques } \\
\text { (Excel) après dépouillement. } \\
\text { (données réelles ou données } \\
\text { simulées) } \\
\text { Alimenter l'outil ainsi construit par } \\
\text { des informations réellement } \\
\text { recueillies ou par des informations } \\
\text { simulées. }\end{array}$ \\
\hline [Prod4A/B] & Individuel & $\begin{array}{l}31 \\
\text { janvier }\end{array}$ & Analyse d'un tableau [Doc9] \\
\hline $\begin{array}{l}{[\operatorname{Prod} 5 \mathrm{~A}]} \\
{[\operatorname{Prod} 5 \mathrm{~B}]}\end{array}$ & $\begin{array}{l}\text { Un par } \\
\text { groupe }\end{array}$ & \begin{tabular}{|l}
31 \\
janvier
\end{tabular} & $\begin{array}{l}\text { Protocole de traitement (Word) } \\
\text { Croisement et ordonnancement des } \\
\text { informations recueillies }\end{array}$ \\
\hline [Prod6A/B] & $\begin{array}{l}\text { Un par } \\
\text { groupe }\end{array}$ & $\begin{array}{l}\text { mars } \\
2007\end{array}$ & $\begin{array}{l}\text { Communication des traitements } \\
\text { annoncés }\end{array}$ \\
\hline $\begin{array}{l}{[\operatorname{Prod} 7 \mathrm{~A}]} \\
{[\operatorname{Prod} 7 \mathrm{~B}]}\end{array}$ & Individuel & $\begin{array}{l}\text { Juin } \\
2007\end{array}$ & $\begin{array}{l}\text { Évaluation finale } 2 \mathrm{~h} \text { par écrit à } \\
\text { partir d'une situation problème } \\
\text { analogue à [Doc10]. Tout } \\
\text { document sera autorisé } \\
\text { Évaluation finale } 2 \mathrm{~h} \text { (à voir) }\end{array}$ \\
\hline
\end{tabular}

\section{V. évaluation :}

La note finale de méthodologie sera la moyenne des 8 notes pondérées : 


\begin{tabular}{|c|c|c|c|c|}
\hline $\begin{array}{l}{[\operatorname{Prod} 1 \mathrm{~A}] \quad[\operatorname{Prod} 2 \mathrm{~A}]} \\
{[\operatorname{Prod} 3 \mathrm{Aa}]} \\
{[\operatorname{Prod} 3 \mathrm{Ab}]}\end{array}$ & [Prod4A/B] & {$[\operatorname{Prod} 5 \mathrm{~A}]$} & [Prod6A/B] & {$[\operatorname{Prod} 7 \mathrm{~A}]$} \\
\hline $12,5 \%$ & \multirow{3}{*}{$12,5 \%$} & $7,5 \%$ & \multirow{3}{*}{$12,5 \%$} & $17,5 \%$ \\
\hline $\begin{array}{l}{[\operatorname{Prod} 1 \mathrm{~B}] \quad[\operatorname{Prod} 2 \mathrm{~B}]} \\
{[\operatorname{Prod} 3 \mathrm{Ba}]} \\
{[\operatorname{Prod} 3 \mathrm{Bb}]}\end{array}$ & & [Prod5B] & & [Prod7B] \\
\hline $12,5 \%$ & & $7,5 \%$ & & $17,5 \%$ \\
\hline
\end{tabular}

\section{Sources et Ressources}

\begin{tabular}{|c|c|}
\hline [Doc1] & $\begin{array}{l}\text { Sites pour des compléments dans le domaine de la } \\
\text { statistique : } \\
\text { http://www.agro-montpellier.fr/cnam-lr/statnet/ réalisé } \\
\text { conjointement par le CNAM et l'Agro-Montpellier et } \\
\text { présenté par Gilbert Saporta. } \\
\text { http://www.univ-lr.fr/formations/idea/duCultureMath } \\
\text { statistiques/ } \\
\text { réalisé conjointement par l'IUFM Poitou-Charentes et } \\
\text { le Département de mathématiques de l'Université de La } \\
\text { Rochelle }\end{array}$ \\
\hline [Doc2] & $\begin{array}{l}\text { Cours Méthodes quantitatives licence, ouvrage et CD } \\
\text { Rom }\end{array}$ \\
\hline [Doc3] & $\begin{array}{l}\text { Compléments du cours Méthodes quantitatives : Tester } \\
\text { des hypothèses. }\end{array}$ \\
\hline [Doc4] & $\begin{array}{l}\text { ParadoxeSimpson.pdf «Liaison entre deux facteurs. } \\
\text { Paradoxe de Simpson » texte de L. Carter, Univ. Paris } \\
\text { X - Nanterre et J-L. Piednoir IG }\end{array}$ \\
\hline [Doc5] & $\begin{array}{l}\text { Présentation d'un test d'adéquation : } \\
\text { ADEQUATION.pdf }\end{array}$ \\
\hline [Doc6] & $\begin{array}{l}\text { Guide d'aide à l'élaboration du dossier } \\
\text { méthodologique. J-C Régnier } \\
\text { Licence }(2004 / 2005): \text { http://pagesperso-orange.fr/jean- } \\
\text { claude.regnier/joao claudio/methode/dossierm1.htm }\end{array}$ \\
\hline [Doc7] & $\begin{array}{l}\text { Complément sur quelques mesures d'association: } \\
\text { MesuresAssociation.pdf }\end{array}$ \\
\hline [Doc8] & $\begin{array}{l}\text { Yanakou Koffiwai Gbati Climat affectif familial et } \\
\text { réussite scolaire : étude auprès d'élèves de cours } \\
\text { moyens première année à Lomé REVUE DE } \\
\text { PSYCHOLOGIE DE L'EDUCATION 2001 - TOME } 1 \\
\text { I.S.S.N. 1279-5097 PUFR - http://www.univ-tours.fr } \\
\text { /editions/som56.htm }\end{array}$ \\
\hline [Doc9] & $\begin{array}{l}\text { Présentation sous PowerPoint d'un des tableaux de } \\
\text { l'article de Yanakou Koffiwai Gbati. } \\
\text { TABLEAUXREVUEPSYCHO1.PPT }\end{array}$ \\
\hline
\end{tabular}


directeur de thèse à l'ED 485 EPIC. Ses axes de recherche s'inscrivent dans les champs de didactiques et apprentissages, et de la statistique, en particulier celui de l'Analyse statistique implicative. Il est aussi très impliqué dans les relations internationales scientifiques franco-brésiliennes.

Université Lumière Lyon 2, 86 rue Pasteur, 69365 Lyon cedex 07

Courriel : jean-claude.regnier@univ-lyon2.fr

Annick PRADEAU est éducatrice spécialisée (problématiques relationnelles intrafamiliales) et étudiante en MARDIF (MAster 2 Recherche à DIstance Francophone en sciences de l'éducation-Université de Rouen) au sein du dispositif Campus numérique FORSE. Durant l'année 2008-2009, elle a réalisé un travail de recherche sur les interactions sociales en formation universitaire en ligne dans le cadre du mémoire de Master1 en sciences de l'éducation de l'Université Lumière Lyon 2

Courriel : annick.pradeau@numericable.com

1 FORSE : FOrmations et Ressources en Sciences de l'Education, consortium réunissant le CNED, l’Université de Rouen et l'Université Lumière Lyon 2. En ligne : http://www.sciencedu.org/

$\stackrel{2}{2}$ Il s'agit de Jean-Claude Régnier co-auteur de cet article et co-fondateur du dispositif FAD - FORSE.

Référence de l'article :

Jean-Claude REGNIER, Annick PRADEAU, Des interactions sociales en formation universitaire à distance, Une approche microsociologique exploratoire et inférentielle, Revue STICEF, Volume 16, 2009, ISSN : 1764-7223, mis en ligne le 26/04/2010, http://sticef.org

(C) Revue Sciences et Technologies de 1'Information et de la Communication pour 1'Éducation et la Formation, 2009 